REVISIONES

Rev Obstet Ginecol Venez. 2021; 81 (4): 377-389.

https://doi.org/10.51288/00810410

\title{
Suplementación de vitamina D en embarazadas y en mujeres con patologías ginecológicas
}

\author{
Jorly Mejia-Montilla, ${ }^{1}$ (D) Eduardo Reyna-Villasmil, ${ }^{2}$ (D) María Albán-Andrade, ${ }^{3}$ \\ Mariela Meza- Lozada, ${ }^{4}$ iD María Cevallos-Rodríguez, ${ }^{5}$ (D) Angélica Manzano-Solís. ${ }^{4}$
}

\begin{abstract}
RESUMEN
Aparte de los efectos de la vitamina $D$ en el mantenimiento de la homeostasis del calcio y promoción de la mineralización ósea, existen pruebas que sugieren que modula procesos reproductivos. Cerca del $70 \%$ de los adultos tienen deficiencias de vitamina D y existe una brecha entre la ingesta dietética recomendada y el suministro deficiente en la población general. Estudios observacionales muestran que la deficiencia de vitamina D es un marcador de riesgo para la reducción de la fertilidad y resultante adversa del embarazo. La evidencia sugiere que podría tener efectos benéficos sobre los parámetros metabólicos, hormonales y celulares del sindrome de ovarios poliquísticos, miomatosis uterina y endometriosis y parece estar asociada con mejoras de los resultados de la fertilización in vitro. El objetivo de esta revisión fue evaluar el papel de la suplementación de vitamina D en embarazadas y en mujeres con patologías ginecológicas.
\end{abstract}

Palabras clave: Vitamina D, Suplementación, Embarazo, Endometriosis, Miomatosis uterina, Infertilidad.

Vitamin D supplementation in pregnant women and in women with gynecological pathologies

\section{SUMMARY}

Apart from the well-known effects of vitamin D in maintaining calcium homeostasis and promoting bone mineralization, there is evidence to suggest that it modulates reproductive processes. About $70 \%$ adults have vitamin $D$ deficiencies and there is a gap between recommended dietary intakes and deficient supply in general population. Observational studies show that vitamin $D$ deficiency is a risk marker for reducing fertility and resulting adverse pregnancy. Evidence suggests that it could have beneficial effects on metabolic, hormonal and cellular parameters of polycystic ovarian syndrome, uterine myomatosis and endometriosis, while it seems to be associated with improvements in outcome of in vitro fertilization. The objective of this review was to evaluate the data of current literature on the role of vitamin D supplementation in pregnant women and in women with gynecological pathologies.

Keywords: Vitamin D, Supplementation, Pregnancy, Endometriosis, Uterine myomatosis, Infertility.

\section{INTRODUCCIÓN}

La deficiencia de vitamina $\mathrm{D}$ (vitD) se considera un problema de salud mundial, ya que gran parte de la

\footnotetext{
${ }^{1}$ Docente de la Facultad de Medicina de La Universidad del Zulia. Maracaibo. Estado Zulia. ${ }^{2}$ Adjunto del servicio de Obstetricia y Ginecología. Hospital Central "Dr. Urquinaona". Maracaibo. Estado Zulia. ${ }^{3}$ Escuela Politécnica Superior de Chimborazo, Riobamba. Ecuador. ${ }^{4}$ Universidad Estatal de Milagro, Milagro. Ecuador. ${ }^{5}$ Escuela Politécnica Superior de Chimborazo, Riobamba. Ecuador. Correo de correspondencia: sippenbauch@gmail.com
}

Forma de citar este artículo: Mejia-Montilla J, Reyna-Villasmil E, Albán-Andrade M, Meza-Lozada M, Cevallos-Rodríguez M, Manzano-Solís A. Suplementación de vitamina D en embarazadas y en mujeres con patologías ginecológicas. Rev Obstet Ginecol Venez. 2021; 81(4):377-389. https://doi. org/10.51288/00810410 población no cumple con los requisitos dietéticos recomendados por las pautas nutricionales (1). Es importante para la salud del sistema musculoesquelético y es eficaz en la prevención y tratamiento del raquitismo y osteomalacia. También puede reducir el número de fracturas en ancianos $(2,3)$.

Varios estudios observacionales han demostrado que la deficiencia de vitD se asocia con diferentes tipos de cáncer, enfermedades cardiovasculares, autoinmunes y neurológicas (3). Por el contrario, los ensayos controlados al azar, han demostrado los efectos de la 


\section{SUPLEMENTACIÓN DE VITAMINA D EN EMBARAZADAS \\ Y EN MUJERES CON PATOLOGÍAS GINECOLÓGICAS}

suplementación en las mujeres con diferentes patologías ginecológicas y durante el embarazo (4). Por lo tanto, se ha sugerido que la deficiencia de vitD puede ser más un marcador de riesgo para enfermedad, que un factor causal de las enfermedades $(4,5)$. En consecuencia, existe controversia científica sobre los posibles efectos extraesqueléticos de la vitD. Sin embargo, debe resaltarse que los metaanálisis de ensayos controlados apoyan la idea de que la suplementación de vitD puede reducir el riesgo de patologías ginecológicas y la resultante obstétrica-perinatal, aunque algunos resultados tienen limitaciones y puede ser prematuro establecer claramente una relación causal. El objetivo de esta revisión fue evaluar los datos de la literatura actual sobre el papel de la suplementación de vitamina $\mathrm{D}$ en embarazadas y en mujeres con patologías ginecológicas.

\section{METABOLISMO DE LA VITAMINA D}

La vitD se sintetiza en la piel durante la exposición a radiación ultravioleta. Menos del $10 \%$ proviene de fuentes dietéticas en ausencia de nutrientes o uso de suplementos. La pro-hormona se transforma en su forma metabólicamente activa en hígado y riñones. Es liposoluble y tiene dos formas principales: colecalciferol (vitD3) y ergocalciferol (vitD2). El colecalciferol surge cuando el ultravioleta B (que tiene una longitud de onda de 290-315 nm) convierte al 7-dehidrocolesterol en los queratinocitos epidérmicos y fibroblastos dérmicos en pro-vitD, que luego se transforma en vitD3. Esta forma está presente en alimentos de origen animal y algunos suplementos. Este proceso de conversión es eficaz y se estima que la exposición breve diaria al sol de la superficie de las manos y cara es similar a una ingesta de 200 unidades de vitD (6). Sin embargo, la duración de la exposición diaria necesaria para obtener el equivalente entre luz solar y suplementación es difícil de determinar y depende del tipo de piel, latitud, estación y hora del día $(7,8)$. La exposición prolongada a la luz solar no produce cantidades tóxicas de vitD3 debido a fotoconversión a metabolitos inactivos. Además, la luz solar induce la producción de melanina, que reduce la producción de vitD3 en piel (9).

La vitD es un nutriente esencial y su efecto pleiotrópico afecta la salud humana. Los alimentos con altas concentraciones incluyen la leche y los granos. Otras fuentes dietéticas incluyen: aceites de hígado de pescado y huevos enriquecidos con la vitamina (10).

\section{EPIDEMIOLOGÍA DE LA DEFICIENCIA DE VITAMINA D}

La investigación epidemiológica sugiere que el déficit de VitD3 es de alrededor del $90 \%$ en la población mundial. A pesar de las diversas directrices sobre la suplementación, el problema de la deficiencia de vitD todavía es común e incluye a las mujeres embarazadas y en edad fértil. La deficiencia se considera como la concentración de 25-hidroxivitamina $[25(\mathrm{OH})$ vitD; forma principal de circulación de vitD] menor a 20 $\mathrm{ng} / \mathrm{mL}(<50 \mathrm{nmol} / \mathrm{L})$, las concentraciones subóptimas en valores de $20-30 \mathrm{ng} / \mathrm{mL}(50-75 \mathrm{nmol} / \mathrm{L})$, mientras que la concentración $30-50 \mathrm{ng} / \mathrm{mL}(75-125 \mathrm{nmol} / \mathrm{L}) \mathrm{se}$ consideraron como normales para lograr los efectos pleiotrópicos (10).

No existen datos exactos sobre las concentraciones de VitD en la población venezolana $(11,12)$. Algunos estudios han evaluado los valores de acuerdo a las concentraciones de $25(\mathrm{OH})$ vitD en adultos en áreas urbanas en diferentes países. Un estudio demostró que casi el $90 \%$ de los sujetos tenían deficiencia moderada o grave, con concentraciones de 25(OH) vitD de 20-30 $\mathrm{ng} / \mathrm{mL}$, que corresponde con los datos de la literatura. Estos datos sugieren que las concentraciones de vitD son insuficientes en adultos, lo que debería llevar a planificar una estrategia preventiva que abarque a la mayoría de la sociedad (13). 


\section{SUPLEMENTACIÓN CON VITAMINA D DURANTE EL EMBARAZO}

La deficiencia de vitD puede afectar a más del $50 \%$ de las embarazadas (14-16). Esta tiene un papel importante al inicio del embarazo, ya que regula la transcripción de genes responsables de la implantación, invasión del trofoblasto y angiogénesis (17). Por medio de su acción inmunomoduladora, también modifica la relación de las citocinas Th2/Th1. Varios hallazgos indican la relación entre la deficiencia de vitD, aparición de complicaciones del embarazo y trastornos del desarrollo fetal.

También se debe prestar atención a la relación entre las concentraciones apropiadas de vitD durante el período fetal e infancia temprana y la salud en la edad adulta. Los procesos de metilación del genoma en el período fetal pueden ser esenciales para la expresión génica y funciones corporales en la vida adulta. Los cambios epigenéticos que conducen al silencio o activación genética tienen como objetivo adaptarse a condiciones ambientales cambiantes. Sin embargo, en el caso de condiciones desfavorables, como la restricción de crecimiento intrauterino fetal, puede existir una programación persistente de procesos metabólicos e inmunológicos que conduzcan a mayor riesgo de enfermedades crónicas (18). La deficiencia de vitD es un factor de riesgo significativo para el desarrollo de las complicaciones relacionadas con preeclampsia y diabetes gestacional (19).

Estudios epidemiológicos indican que estas complicaciones obstétricas se asocian con mayor incidencia de obesidad, diabetes, enfermedades cardiovasculares y trastornos neurológicos y de comportamiento en la edad adulta $(20,21)$. Estudios en embarazadas demostraron que la concentración de vitD en sangre del cordón umbilical estaba relacionada por la concentración en sangre materna $(22,23)$. El receptor de vitD(VDR) desempeña un papel importante en la activación de la respuesta inmune, así como en la expresión/inhibición de la transcripción de al menos 291 genes (24). Existe evidencia de correlación negativa del índice de calcidiol libre materno con la metilación del receptor RXRA (cofactor del VDR) en la sangre del cordón umbilical (25). La hipermetilación del receptor RXRA al nacer se asoció con menor mineralización ósea en el cuarto año de vida. Estudios al azar demostraron que la suplementación con 200 UI de vitD durante el período preconcepcional redujo la metilación en sangre del cordón umbilical del receptor IGF2R en niñas y GT2L en niños (26).

Los trastornos de la implantación en el embarazo temprano son decisivos en el desarrollo de complicaciones en la segunda mitad del embarazo, como la preeclampsia. En estudios realizados en secciones de sincitiotrofoblasto de embarazos complicados con preeclampsia, se observó inhibición de la producción de calcitriol asociado a la reducción de la expresión del gen de la $1 \alpha$ hidroxilasa comparado con embarazos normales. La razón de esto no está clara, especialmente porque no se han encontrado alteraciones en la relación fosfato/calcio y en la secreción de paratohormona en preeclámpticas (27). Las bajas concentraciones de vitD también se asocian a mayor riesgo de preeclampsia antes de la semana 34 de embarazo. En las embarazadas que desarrollaron preeclampsia temprana, además tenían concentraciones más bajas de $25(\mathrm{OH})$ vitD aquellas pacientes con restricción del crecimiento intrauterino comparado con el subgrupo de neonatos con peso normal. El percentil del peso al nacer mostró correlación positiva con la concentración materna de $25(\mathrm{OH})$ vitD (28). También en las embarazadas sin preeclampsia se encontró relación entre los valores más bajos de vitD materna y el bajo peso al nacer.

Además de la preeclampsia, el mayor problema de la medicina perinatal es el parto pretérmino. El análisis de mujeres embarazadas indicó que aquellas con valores de $25(\mathrm{OH})$ vitD superior a $40 \mathrm{ng} / \mathrm{mL}$ tenían un riesgo $57 \%$ menor de parto pretérmino 


\section{SUPLEMENTACIÓN DE VITAMINA D EN EMBARAZADAS \\ Y EN MUJERES CON PATOLOGÍAS GINECOLÓGICAS}

en comparación con aquellas cuya concentración no superaba $20 \mathrm{ng} / \mathrm{mL}$ (29). Una revisión sistemática de estudios aleatorios sobre suplementación con vitD durante el embarazo demostró que esta redujo el riesgo tanto de preeclampsia como de parto pretérmino (30). Teniendo en cuenta la relación entre bajo peso al nacer y mayor riesgo de enfermedades metabólicas en la vida adulta, un efecto importante de la suplementación en embarazadas es reducir el riesgo de neonatos con bajo peso al nacer.

En estudios observacionales de cohorte, también se encontraron concentraciones más bajas de $25(\mathrm{OH})$ vitD en pacientes con diabetes gestacional. Algunos estudios sugieren un efecto metabólico positivo de la ingesta de vitD en embarazadas con disminución de riesgo de diabetes gestacional. Los resultados de un estudio europeo demostraron que la suplementación disminuye el riesgo de diabetes gestacional y sus complicaciones (31).

Hasta el momento, no se ha demostrado efecto pernicioso de la suplementación con vitD en la salud materno-fetal. La suplementación se considera una medida de prevención segura de las complicaciones del embarazo y del período perinatal.

\section{SUPLEMENTACIÓN DE VITAMINA D EN PATOLOGÍAS GINECOLÓGICAS}

\section{Endometriosis}

Existe evidencia de la presencia de VDR y enzimas metabolizadoras de vitD en endometrio y miometrio humano. Uno de los primeros estudios de casos y controles encontró mayores concentraciones séricas de vitD en mujeres con endometriosis (32). No obstante, estudios posteriores no lograron demostrar diferencias en las concentraciones séricas de pacientes con endometriosis, pero estas pacientes sobreexpresan VDR y $1 \alpha$-hidroxilasa en los focos de endometriosis peritoneal y endometrio comparado con los controles sanos (33). Se ha propuesto que existe hipersensibilidad inmunológica secundaria a estimulación excesiva de las células inmunes por las concentraciones peritoneales elevadas de vitD, produciendo un impacto local dentro de implantes endometriales (34). Otro estudio sobre el análisis proteómico del suero extraído de pacientes con endometriosis demostró mayor expresión de la proteína de unión a la vitD (VDBP) (35).

Las críticas a estos trabajos indican que las observaciones de concentraciones séricas elevadas de vitD y su sobreexpresión en focos ectópicos endometriósicos pueden ser el resultado de selección inadecuada de grupos controles, que incluían mujeres con miomatosis uterina e infertilidad idiopática o enfermedades caracterizadas por deficiencia significativa de vitD (36). Además, estos estudios se caracterizaron por ser retrospectivos, con muestras pequeñas y heterogéneos.

Contrario a las observaciones de informes previos, un estudio de cohorte prospectivo analizó la relación entre la ingesta de vitD, sus concentraciones séricas y el diagnóstico de la endometriosis por laparoscopia. El estudio confirmó que la concentración sérica se relacionaba en forma inversa con la presencia de endometriosis, mientras que las mujeres con las concentraciones más elevadas tenían menor riesgo (24\%) de desarrollar endometriosis comparado con el grupo de concentraciones más bajas. Además, las mujeres en el grupo con la ingesta más alta de vitD en los alimentos (el quintil más alto) tenían un riesgo $21 \%$ menor de diagnóstico de endometriosis que las pacientes en el quintil más bajo (37). Estos hallazgos fueron confirmados por estudios posteriores con modelos animales de endometriosis, en los cuales la administración de vitD o del receptor selectivo (elocalcitol) inhibe el desarrollo de la endometriosis y reduce los marcadores de inflamación en la cavidad peritoneal $(38,39)$. 
Un estudio al azar, doble ciego y controlado con placebo investigó la eficacia de la suplementación de vitD en dismenorrea primaria (40). Este demostró la efectividad de una dosis única de 300000 UI administrada antes de la menstruación, logrando una reducción significativa durante dos meses de observación. También se logró demostrar la correlación inversa entre las concentraciones séricas de vitD y la intensidad del dolor. El mecanismo de analgesia probable consiste en inhibir la producción de prostaglandinas y estimular su inactivación al aumentar la actividad de la hidroxiprostaglandina deshidrogenasa e inhibir la ciclooxigenasa 2 (41).

\section{Leiomiomatosis uterina}

Varios estudios han demostrado que la deficiencia de vitD puede ser un factor de riesgo para el desarrollo de leiomiomatosis uterina $(42,43)$. En un estudio de casos y controles se demostró que las concentraciones séricas eran más bajas en pacientes con miomatosis. Luego de la corrección por índice de masa corporal, la razón de probabilidad para la aparición de miomatosis uterina en mujeres con deficiencia de vitD (menor de $10 \mathrm{ng} / \mathrm{mL}$ ) fue de 2,4 (intervalo de confianza $95 \%$, $1,2-4,9)(44)$.

El efecto antiproliferativo de la vitD en células del miometrio humanas y células derivadas de los miomas ha sido documentado en varios estudios in vitro (45-48). La vitD es un inhibidor de Wnt $4 / \beta$ catenina e inhibe la transducción de la vía mTOR, que desempeña un papel importante en la patogénesis de los miomas. El potencial efecto terapéutico durante la formación y/o crecimiento de los miomas también se ha documentado en modelos animales in vivo (49).

\section{Síndrome de ovarios poliquísticos}

El síndrome de ovarios poliquísticos (SOPQ) es una endocrinopatía común que afecta aproximadamente $6 \%$
- $12 \%$ de las mujeres en edad fértil, con manifestaciones heterogéneas. Además de sangrado menstrual irregular, ovarios morfológicamente poliquísticos, hiperandrogenismo y/o hiperandrogenemia, un subgrupo presenta obesidad y sobrepeso (20\% - $85 \%)$, insulinorresistencia y otros trastornos metabólicos.

Un metaanálisis que seleccionó estudios de cohortes encontró que el aumento en el índice de masa corporal de $1 \mathrm{Kg} / \mathrm{m} 2$ se asoció con disminución de las concentraciones de vitD en más de $1 \%$. Al mismo tiempo, un aumento del $10 \%$ del índice de masa corporal se asocia con disminución en la concentración sérica en más de $4 \%$ (50). Estas observaciones proporcionan evidencia de que la obesidad y el sobrepeso, que coexisten en las mujeres con SOPQ, conducen a deficiencia de vitD. El principal mecanismo patológico de este fenómeno es el secuestro de vitD por el tejido adiposo.

No existen datos que indiquen la posible relación entre las bajas concentraciones de vitD y la patogénesis del SOPQ, especialmente en mujeres con índice de masa corporal normal $(51,52)$. Un estudio demostró correlación entre las concentraciones de vitD con las concentraciones de andrógenos y la relación hormona luteinizante/hormona foliculoestimulante (53). Sin embargo, la relación con otros parámetros endocrinos aún no se ha confirmado $(54,55)$. En varios estudios observacionales se ha confirmado la relación de las bajas concentraciones séricas de vitD y el desarrollo de insulinorresistencia, tolerancia alterada a la glucosa, dislipidemia, obesidad e hipertensión (51-57).

La vitD puede tener efectos benéficos sobre las concentraciones de insulina al estimular la expresión de receptores de insulina y mejorar el metabolismo de la glucosa. En el promotor del gen de insulina humana existe un elemento sensible a la estimulación por la vitD, que activa la transcripción del gen. Además, regula el suministro de calcio extra- e intracelular que 


\section{SUPLEMENTACIÓN DE VITAMINA D EN EMBARAZADAS \\ Y EN MUJERES CON PATOLOGÍAS GINECOLÓGICAS}

modula la secreción de insulina. Los polimorfismos en el gen del VDR pueden predisponer al desarrollo del SOPQ (58). Existe evidencia de asociación de los polimorfismos del VDR (CDX-2, Taq I, Bsm-I) y trastornos de la insulina, hiperandrogenismo, concentraciones elevadas de hormona luteinizante y disminución de las concentraciones de globulina fijadora de hormonas sexuales en mujeres con SOPQ $(59,60)$.

La evidencia de la eficacia terapéutica de la suplementación de vitD en el tratamiento del SOPQ proviene de estudios de intervención. La suplementación con 50000 UI 1 a 2 veces por semana junto a $1500 \mathrm{mg}$ de calcio, durante seismeses, en mujeres premenopáusicas con hiperandrogenismo, llevó a normalización del ciclo menstrual en la mayoría de las pacientes después de 2 meses de tratamiento (61). Del mismo modo, otro estudio en mujeres con SOPQ que recibieron 20000 UI de colecalciferol semanalmente durante 24 semanas, regularizó la menstruación en el $50 \%$ de las participantes, acompañado de cambios benéficos en el metabolismo glucémico, sin cambios significativos en el índice de masa corporal (56). Otros estudios demostraron que la suplementación mejora significativamente la insulinorresistencia y el perfil lipídico, sin afectar el perfil endocrino en mujeres con SOPQ (62-64). Además, la suplementación con vitD puede reparar la foliculogénesis ovárica y conducir a una ovulación espontánea (65).

El efecto benéfico de la vitD sobre trastornos metabólicos en mujeres con SOPQ no ha podido ser confirmado por estudios al azar y doble ciego. Un ensayo controlado aleatorio en un grupo de mujeres con SOPQ tratados con vitD (50 $000 \mathrm{UI} /$ día durante 2 meses) demostró mejora del perfil lipídico, sin modificaciones en los marcadores inflamatorios (66). Otros dos estudios que utilizaron $12000 \mathrm{UI} /$ día de vitD durante 12 semanas (67) y 4000 UI/día durante 6 meses y metformina (68), no demostraron cambios en la insulinorresistencia, a pesar de la normalización de la presión arterial. En dos estudios, uno prospectivo de cohortes (69) y un ensayo controlado aleatorio (70) se observaron efectos benéficos de la suplementación con vitD sobre la estimulación de la ovulación con citrato de clomífero y aumento de la tasa de embarazos con técnicas de fertilización in vitro en mujeres infértiles con SOPQ $(70,71)$.

\section{SUPLEMENTACIÓN DE VITAMINA D EN MEDICINA REPRODUCTIVA}

Reserva ovárica

Un estudio transversal realizado en mujeres premenopáusicas con menstruaciones regulares encontró correlación positiva entre las concentraciones séricas de vitD y de hormona antimülleriana, después de la corrección por índice de masa corporal, hábito tabáquico, raza e insulinorresistencia. Esta relación también fue observada en la subpoblación de mujeres mayores de 40 años, lo que sugiere que la deficiencia de vitD puede estar asociada con disminución significativa de la reserva ovárica, especialmente al final del período reproductivo (71).

Infertilidad y reproducción asistida

Existen estudios in vitro e in vivo que describen la relación entre deficiencia de vitD y la disfunción ovárica. En modelos humanos y animales, se ha demostrado que regula la expresión de receptores para hormona foliculoestimulante y antimülleriana, controlando la foliculogénesis y la diferenciación de las células de la granulosa. También aumenta la expresión de genes para enzimas claves para la esteroidogénesis y estimula la producción de progesterona y estrógenos, controlando el desarrollo adecuado del cuerpo lúteo (72). Los ratones que carecen de un gen activo para VDR tienen alteraciones de la foliculogénesis, ausencia de ovulaciones y útero hipoplásico. La dieta 
baja en vitD, que conduce a deficiencia, produce hasta un $70 \%$ de reducción de la fertilidad (73).

Aunque los estudios clínicos en humanos sobre la relación entre deficiencia de vitD, infertilidad, respuesta a la estimulación de la ovulación y efectividad de la fertilización in vitro son escasos, proporcionan datos contradictorios. Existen investigaciones que indican un porcentaje alto $(20 \%-100 \%)$ de deficiencia de vitD en mujeres que se encuentra en programas de fertilización asistida (74-77). Un estudio prospectivo demostró la relación positiva entre las concentraciones de vitD en el líquido folicular y en el suero y la proporción de embarazos logrados durante un programa de fertilización in vitro (51). La mayor tasa de implantación exitosa se obtuvo en el grupo de mujeres cuyas concentraciones de vitD en el líquido folicular estaban en los valores más altos (75).

Un estudio de cohortes retrospectivo demostró que las concentraciones séricas de vitD influyen en la efectividad del programa de fertilización asistida. Se encontró asociación entre disminución lineal en las concentraciones de vitD y la reducción de la tasa de embarazos. Sin embargo, no se observó afectación de la calidad de la estimulación ovárica o de los embriones resultantes (76). Otro estudio retrospectivo posterior demostró que las concentraciones más altas de vitD se relacionaban con mayores tasas de embarazos clínicos y recién nacidos vivos. El porcentaje de embarazos clínicos fue el doble en el grupo de mujeres con concentraciones normales. El diseño de ese estudio permitió probar que la vitD afecta el endometrio sin afectar en forma significativa la respuesta a la estimulación de la ovulación y la calidad del embrión (77).

A pesar de que estos datos sugieren una relación entre la deficiencia de vitD y la falla del programa de fertilidad asistida, otros estudios prospectivos de cohortes no confirman esa asociación $(74,78)$. Además, algunas investigaciones han demostrado efectos negativos de las concentraciones más altas en la calidad de los embriones obtenidos en el programa de fertilización in vitro (78). Tampoco se observaron efectos de la deficiencia en la eficacia de la los procedimientos de fertilización asistida, independientemente de la determinación de la concentración total o biodisponible de vitD (79). Otro estudio retrospectivo que evaluó la transferencia de blastocito encontró una proporción menor de embarazos en mujeres con deficiencia de vitD, considerando aun los factores que interfieren con la probabilidad de embarazo (80). La falta de relación previamente descrita también fue demostrada en un estudio retrospectivo de cohortes posterior (81).

Los hallazgos contradictorios sobre la relación de la deficiencia de vitD con la efectividad de los programas de fertilización asistida son resultado de numerosos factores de confusión, diferentes definiciones de deficiencia de vitD, varias metodologías para su determinación y la transferencia de diferente número de embriones. A pesar de la falta de evidencia y de las recomendaciones para la determinación de las concentraciones de vitD y el papel de la posible suplementación antes del inicio del programa de fertilización in vitro, la mayoría de los datos apuntan hacia una posible relación causal $(36,82)$.

\section{DOSIS PARA LA SUPLEMENTACIÓN DE VITAMINA D}

La fuente de vitD es de origen endógeno (síntesis en la piel) y exógeno (dieta). Las fuentes exógenas incluyen la leche, mantequilla, bebidas derivadas de soja, champiñones y pescado graso (83-85). Las condiciones climáticas y hábitos alimenticios en muchos países pueden causar su deficiencia. La cantidad de vitD que debería aportar la dieta es en promedio de 2,5 4,0 $\mu \mathrm{g} /$ día, lo que representa aproximadamente $3 \%$ - $5 \%$ de las necesidades diarias (86). En la población adulta general, $10 \%-60 \%$ de las personas tienen 


\section{SUPLEMENTACIÓN DE VITAMINA D EN EMBARAZADAS \\ Y EN MUJERES CON PATOLOGÍAS GINECOLÓGICAS}

concentraciones séricas de vitD por debajo de $20 \mathrm{ng} /$ $\mathrm{mL}$. El objetivo de la suplementación es asegurar concentraciones séricas suficientes de $25(\mathrm{OH})$ vitD (87).

Considerando el impacto negativo de la deficiencia de vitD sobre la salud, se debe considerar que la suplementación está indicada en estados de deficiencia confirmada. La creciente cantidad de evidencia de los efectos benéficos fue la razón para elaborar recomendaciones para la prevención de la deficiencia. De acuerdo con las pautas, la dosis diaria de vitD debe estar entre 1500 - 2000 UI para adultos de 18 a 70 años para lograr una concentración sérica constante por encima de $30 \mathrm{ng} / \mathrm{mL}$. Las concentraciones por encima de este valor se consideran las más benéficas (88). Una dosis diaria de 10000 UI se considera aceptable (89).

Debido a la posibilidad de disminuir la insulinorresistencia, es recomendable utilizar la vitD en pacientes con intolerancia a la glucosa y diabetes gestacional. La dosis diaria debe exceder la dosis de suplementación, pero no exceder 10000 UI/día. Para lograr la disminución de la insulinorresistencia se necesitan concentraciones séricas entre 32 y $47 \mathrm{ng} / \mathrm{mL}$ (89). Por otra parte, el uso de vitD en estados de reducción de la fertilidad (ciclos anovulatorios o alteraciones en la duración del ciclo menstrual) se logra con concentraciones séricas que no exceden $30 \mathrm{ng} / \mathrm{ml}$ y dosis diarias inferiores a $4000 \mathrm{UI} /$ día $(90,91)$.

Las embarazadas y puérperas en período de lactancia deben evitar la deficiencia de vitD3 antes del embarazo y usar dosis suplementarias entre 1500 - 2000 UI/día. La suplementación debe iniciarse lo antes posible. Si es posible, se debe monitorear las concentraciones séricas de $25(\mathrm{OH})$ vitD para alcanzar un valor de 30-50 ng/mL (92, 93).

\section{CONCLUSIONES}

La evidencia actual de los estudios clínicos indica que la deficiencia de vitD es muy común en la población general, y especialmente en embarazadas y mujeres con patologías ginecológicas. Estudios observacionales, tanto en animales como en humanos, apuntan fuertemente hacia una asociación con la fertilidad femenina. Los datos indican que podría ser parte de la patogénesis y la prevención de la endometriosis, mientras que la deficiencia se ha relacionado con menores tasas de fertilización in vitro. Además, la suplementación en mujeres con SOPQ mejoró algunos de los trastornos metabólicos y, principalmente, los trastornos reproductivos. No obstante, los resultados de ensayos clínicos controlados aleatorios muestran resultados inconsistentes. La suplementación de vitD puede producir efectos benéficos en embarazadas, pero aún no se tienen conclusiones claras. La suplementación de vitD es económica, bien tolerada y segura. Además, las dosis recomendadas son seguras y aumentan las concentraciones séricas de $25(\mathrm{OH})$ vitD.

\section{REFERENCIAS}

1. Bouillon R. How much vitamin D is needed for healthy bones? J Intern Med. 2017; 282(5):461-464. doi: 10.1111/joim.12677.

2. Kassi E, Adamopoulos C, Basdra EK, Papavassiliou AG. Role of vitamin $\mathrm{D}$ in atherosclerosis. Circulation. 2013; 128(23):2517-2531. doi: 10.1161/ CIRCULATIONAHA.113.002654.

3. GBD 2017 Population and Fertility Collaborators. Population and fertility by age and sex for 195 countries and territories, 1950-2017: a systematic analysis for the Global Burden of Disease Study 2017. Lancet. 2018; 392(10159):1995-2051. doi: 10.1016/S01406736(18)32278-5.

4. Muscogiuri G, Altieri B, de Angelis C, Palomba S, Pivonello R, Colao A, et al. Shedding new light on female fertility: The role of vitamin D. Rev Endocr Metab Disord. 2017; 18(3):273-283. doi: 10.1007/ s11154-017-9407-2. 
5. Simon L, Murphy K, Shamsi MB, Liu L, Emery B, Aston $\mathrm{KI}$, et al. Paternal influence of sperm DNA integrity on early embryonic development. Hum Reprod. 2014; 29(11):2402-2412. doi: 10.1093/humrep/deu228.

6. Holick MF, Hossein-Nezhad A. The D-lemma: narrow-band UV type B radiation versus vitamin D supplementation versus sunlight for cardiovascular and immune health. Am J Clin Nutr. 2017; 105(5):10311032. doi: 10.3945/ajen.117.155713.

7. Terushkin V, Bender A, Psaty EL, Engelsen O, Wang SQ, Halpern AC. Estimated equivalency of vitamin D production from natural sun exposure versus oral vitamin D supplementation across seasons at two US latitudes. J Am Acad Dermatol. 2010; 62(6):929.e1-9. doi: 10.1016/j.jaad.2009.07.028.

8. O'Sullivan F, Laird E, Kelly D, van Geffen J, van Weele M, McNulty H, et al. Ambient UVB Dose and Sun Enjoyment Are Important Predictors of Vitamin D Status in an Older Population. J Nutr. 2017; 147(5):858868. doi: $10.3945 /$ jn. 116.244079 .

9. Holick MF, MacLaughlin JA, Doppelt SH. Regulation of cutaneous previtamin D3 photosynthesis in man: skin pigment is not an essential regulator. Science. 1981; 211(4482):590-593. doi: 10.1126/science.6256855.

10. Hochberg Z, Hochberg I. Evolutionary Perspective in Rickets and Vitamin D. Front Endocrinol (Lausanne). 2019; 10:306. doi: 10.3389/fendo.2019.00306.

11. López Goitia D, Riera Espinoza G, Romano JA, Ramos J, Stanbury G. Déficit de vitamina D en hombres y mujeres obesos en pre-operatorio para cirugía bariátrica: Una Alerta necesaria. Rev Venez Endocrinol Metab [Internet]. 2015 [consultado febrero de 2019]; 13(1):25-52. Disponible en: http://ve.scielo. org/scielo.php?script=sci_arttext\&pid $=$ S 1690 31102015000100004\&lng=es.

12. Querales MI, Mendoza C, Cruces ME, Díaz L, Navarro G, Navas M. Vitamina D en pacientes con síndrome metabólico de la ciudad de Valencia, Venezuela. An Venez Nutr [Internet]. 2013 [consultado marzo de 2019]; 26(2):78-85. Disponible en: http://ve.scielo. org/scielo.php?script=sci_arttext\&pid $=\mathrm{S} 0798$ 07522013000200003\&lng=en.

13. Mendes MM, Darling AL, Hart KH, Morse S, Murphy RJ, Lanham-New SA. Impact of high latitude, urban living and ethnicity on 25-hydroxyvitamin D status: A need for multidisciplinary action? J Steroid Biochem Mol Biol. 2019; 188:95-102. doi: 10.1016/j. jsbmb.2018.12.012.

14. Bomba-Opon DA, Brawura-Biskupski-Samaha
R, Kozlowski S, Kosinski P, Bartoszewicz Z, Bednarczuk $\mathrm{T}$, et al. First trimester maternal serum vitamin D and markers of preeclampsia. J Matern Fetal Neonatal Med. 2014; 27(10):1078-1079. doi: 10.3109/14767058.2013.846318.

15. Bartoszewicz Z, Kondracka A, Krasnodebska-Kiljańska M, Niedźwiedzka B, Popow M, Ambroziak U, et al. Vitamin D insufficiency in healthy pregnant women living in Warsaw. Ginekol Pol. 2013; 84(5):363-367. doi: $10.17772 / \mathrm{gp} / 1590$.

16. Skowrońska-Jóźwiak E, Adamczewski Z, Tyszkiewicz A, Krawczyk-Rusiecka K, Lewandowski K, Lewiński A. Assessment of adequacy of vitamin D supplementation during pregnancy. Ann Agric Environ Med [Internet]. 2014 [consultado febrero de 2019]; 21(1):198-200. Disponible en: http://www.aaem.pl/Assessment-ofadequacy-of-vitamin-D-supplementation-duringpregnancy, $72085,0,2 . h t m l$

17. Ganguly A, Tamblyn JA, Finn-Sell S, Chan SY, Westwood M, Gupta J, et al. Vitamin D, the placenta and early pregnancy: effects on trophoblast function. J Endocrinol. 2018; 236(2):R93-R103. doi: 10.1530/ JOE-17-0491.

18. Tian FY, Marsit CJ. Environmentally Induced Epigenetic Plasticity in Development: Epigenetic Toxicity and Epigenetic Adaptation. Curr Epidemiol Rep. 2018; 5(4):450-460. doi: 10.1007/s40471-0180175-7.

19. Roth DE, Leung M, Mesfin E, Qamar H, Watterworth J, Papp E. Vitamin D supplementation during pregnancy: state of the evidence from a systematic review of randomised trials. BMJ. 2017; 359:j5237. doi: 10.1136/ bmj.j5237.

20. Jazwiec PA, Sloboda DM. Nutritional adversity, sex and reproduction: 30 years of $\mathrm{DOHaD}$ and what have we learned? J Endocrinol. 2019; 242(1):T51-T68. doi: 10.1530/JOE-19-0048.

21. Krishnaveni GV, Yajnik CS. Developmental origins of diabetes-an Indian perspective. Eur J Clin Nutr. 2017; 71(7):865-869. doi: 10.1038/ejen.2017.87.

22. O’Brien KO, Li S, Cao C, Kent T, Young BV, Queenan RA, et al. Placental CYP27B1 and CYP24A1 expression in human placental tissue and their association with maternal and neonatal calcitropic hormones. J Clin Endocrinol Metab. 2014; 99(4):1348-1356. doi: 10.1210/jc.2013-1366.

23. Aghajafari F, Field CJ, Rabi D, Kaplan BJ, Maggiore JA, O'Beirne M, et al.; APrON Study Team. Plasma 3-Epi-25-Hydroxycholecalciferol Can Alter the 


\section{SUPLEMENTACIÓN DE VITAMINA D EN EMBARAZADAS \\ Y EN MUJERES CON PATOLOGÍAS GINECOLÓGICAS}

Assessment of Vitamin D Status Using the Current Reference Ranges for Pregnant Women and Their Newborns. J Nutr. 2016; 146(1):70-75. doi: 10.3945/ jn.115.220095.

24. Rovner AJ, Stallings VA, Rutstein R, Schall JI, Leonard MB, Zemel BS. Effect of high-dose cholecalciferol (vitamin D3) on bone and body composition in children and young adults with HIV infection: a randomized, double-blind, placebo-controlled trial. Osteoporos Int. 2017; 28(1):201-209. doi: 10.1007/s00198-0163826-X.

25. Curtis EM, Krstic N, Cook E, D’Angelo S, Crozier SR, Moon RJ, et al.; MAVIDOS Trial Group. Gestational Vitamin D Supplementation Leads to Reduced Perinatal RXRA DNA Methylation: Results From the MAVIDOS Trial. J Bone Miner Res. 2019; 34(2):231240. doi: 10.1002/jbmr.3603.

26. Cooper WN, Khulan B, Owens S, Elks CE, Seidel $\mathrm{V}$, Prentice AM, et al. DNA methylation profiling at imprinted loci after periconceptional micronutrient supplementation in humans: results of a pilot randomized controlled trial. FASEB J. 2012; 26(5):1782-1790. doi: 10.1096/fj.11-192708.

27. Palaniswamy S, Williams D, Järvelin MR, Sebert S. Vitamin D and the Promotion of Long-Term Metabolic Health from a Programming Perspective. Nutr Metab Insights. 2016; 8(Suppl 1):11-21. doi: 10.4137/NMI. S29526.

28. Robinson CJ, Wagner CL, Hollis BW, Baatz JE, Johnson DD. Association of maternal vitamin D and placenta growth factor with the diagnosis of early onset severe preeclampsia. Am J Perinatol. 2013; 30(3):167172. doi: 10.1055/s-0032-1322514.

29. Wagner CL, Baggerly C, McDonnell S, Baggerly KA, French CB, Baggerly L, et al. Post-hoc analysis of vitamin $\mathrm{D}$ status and reduced risk of preterm birth in two vitamin D pregnancy cohorts compared with South Carolina March of Dimes 2009-2011 rates. J Steroid Biochem Mol Biol. 2016; 155(Pt B):245-251. doi: 10.1016/j.jsbmb.2015.10.022.

30. Antonakou A. Vitamin D supplementation for women during pregnancy. Women Birth. 2018; 31(4):e286. doi: 10.1016/j.wombi.2017.11.001.

31. Jelsma JG, van Poppel MN, Galjaard S, Desoye G, Corcoy R, Devlieger R, et al. DALI: Vitamin D and lifestyle intervention for gestational diabetes mellitus (GDM) prevention: an European multicentre, randomised trial - study protocol. BMC Pregnancy Childbirth. 2013; 13:142. doi: 10.1186/1471-2393-13142.
32. Somigliana E, Panina-Bordignon P, Murone S, Di Lucia P, Vercellini P, Vigano P. Vitamin D reserve is higher in women with endometriosis. Hum Reprod. 2007; 22(8):2273-2278. doi: 10.1093/humrep/dem142.

33. Agic A, Xu H, Altgassen C, Noack F, Wolfler MM, Diedrich K, Friedrich M, et al. Relative expression of 1,25-dihydroxyvitamin D3 receptor, vitamin D 1 alphahydroxylase, vitamin D 24-hydroxylase, and vitamin D 25-hydroxylase in endometriosis and gynecologic cancers. Reprod Sci. 2007; 14(5):486-497. doi: 10.1177/1933719107304565.

34. Sayegh L, Fuleihan Gel-H, Nassar AH. Vitamin D in endometriosis: a causative or confounding factor? Metabolism. 2014; 63(1):32-41. doi: 10.1016/j. metabol.2013.09.012.

35. Hwang JH, Wang T, Lee KS, Joo JK, Lee HG. Vitamin $\mathrm{D}$ binding protein plays an important role in the progression of endometriosis. Int J Mol Med. 2013; 32(6):1394-1400. doi: 10.3892/ijmm.2013.1506.

36. Chu J, Gallos I, Tobias A, Tan B, Eapen A, Coomarasamy A. Vitamin D and assisted reproductive treatment outcome: a systematic review and meta-analysis. Hum Reprod. 2018; 33(1):65-80. doi: 10.1093/humrep/dex326.

37. Harris HR, Chavarro JE, Malspeis S, Willett WC, Missmer SA. Dairy-food, calcium, magnesium, and vitamin D intake and endometriosis: a prospective cohort study. Am J Epidemiol. 2013; 177(5):420-430. doi: 10.1093/aje/kws247.

38. Mariani M, Viganò $\mathrm{P}$, Gentilini $\mathrm{D}$, Camisa $\mathrm{B}$, Caporizzo E, Di Lucia P, et al. The selective vitamin $\mathrm{D}$ receptor agonist, elocalcitol, reduces endometriosis development in a mouse model by inhibiting peritoneal inflammation. Hum Reprod. 2012; 27(7):2010-2019. doi: 10.1093/humrep/des150.

39. Abbas MA, Taha MO, Disi AM, Shomaf M. Regression of endometrial implants treated with vitamin D3 in a rat model of endometriosis. Eur J Pharmacol. 2013; 715(13):72-75. doi: 10.1016/j.ejphar.2013.06.016.

40. Lasco A, Catalano A, Benvenga S. Improvement of primary dysmenorrhea caused by a single oral dose of vitamin D: results of a randomized, double-blind, placebo-controlled study. Arch Intern Med. 2012; 172(4):366-367. doi: 10.1001/archinternmed.2011.715.

41. Abu El Maaty MA, Wölfl S. Vitamin D as a Novel Regulator of Tumor Metabolism: Insights on Potential Mechanisms and Implications for Anti-Cancer Therapy. Int J Mol Sci. 2017; 18(10):2184. doi: 10.3390/ ijms 18102184 .

42. Ciebiera M, Włodarczyk M, Słabuszewska-Jóźwiak A, Nowicka G, Jakiel G. Influence of vitamin D and 
transforming growth factor $\beta 3$ serum concentrations, obesity, and family history on the risk for uterine fibroids. Fertil Steril. 2016; 106(7):1787-1792. doi: 10.1016/j. fertnstert.2016.09.007.

43. Sabry M, Halder SK, Allah AS, Roshdy E, Rajaratnam V, Al-Hendy A. Serum vitamin D3 level inversely correlates with uterine fibroid volume in different ethnic groups: a cross-sectional observational study. Int J Womens Health. 2013; 5:93-100. doi: 10.2147/IJWH. S38800.

44. Paffoni A, Somigliana E, Vigano' P, Benaglia L, Cardellicchio L, Pagliardini L, et al. Vitamin D status in women with uterine leiomyomas. J Clin Endocrinol Metab. 2013; 98(8):E13741378. doi: 10.1210/jc.2013-1777.

45. Bläuer M, Rovio PH, Ylikomi T, Heinonen PK. Vitamin $\mathrm{D}$ inhibits myometrial and leiomyoma cell proliferation in vitro. Fertil Steril. 2009; 91(5):1919-1925. doi: 10.1016/j.fertnstert.2008.02.136.

46. Al-Hendy A, Diamond MP, Boyer TG, Halder SK. Vitamin D3 Inhibits Wnt/ $\beta$-Catenin and mTOR Signaling Pathways in Human Uterine Fibroid Cells. J Clin Endocrinol Metab. 2016; 101(4):1542-1551. doi: 10.1210/jc.2015-3555.

47. Halder SK, Goodwin JS, Al-Hendy A. 1,25-Dihydroxyvitamin D3 reduces TGF-beta3induced fibrosis-related gene expression in human uterine leiomyoma cells. J Clin Endocrinol Metab. 2011; 96(4):E754-762. doi: 10.1210/jc.2010-2131.

48. Sharan C, Halder SK, Thota C, Jaleel T, Nair S, Al-Hendy A. Vitamin D inhibits proliferation of human uterine leiomyoma cells via catechol-Omethyltransferase. Fertil Steril. 2011; 95(1):247-253. doi: 10.1016/j.fertnstert.2010.07.1041.

49. Halder SK, Sharan C, Al-Hendy A. 1,25-dihydroxyvitamin D3 treatment shrinks uterine leiomyoma tumors in the Eker rat model. Biol Reprod. 2012; 86(4):116. doi: 10.1095/biolreprod.111.098145.

50. Vimaleswaran KS, Berry DJ, Lu C, Tikkanen E, Pilz S, Hiraki LT, et al. Causal relationship between obesity and vitamin D status: bi-directional Mendelian randomization analysis of multiple cohorts. PLoS Med. 2013; 10(2):e1001383. doi: 10.1371/journal.pmed.1001383.

51. Güdücü N, Görmüş U, Kutay SS, Kavak ZN, Dünder I. 25-Hydroxyvitamin D levels are related to hyperinsulinemia in polycystic ovary syndrome. Gynecol Endocrinol. 2014; 30(8):557-560. doi: 10.3109/09513590.2014.910189.

52. Azadi-Yazdi M, Nadjarzadeh A, Khosravi-Boroujeni H, Salehi-Abargouei A. The Effect of Vitamin D
Supplementation on the Androgenic Profile in Patients with Polycystic Ovary Syndrome: A Systematic Review and Meta-Analysis of Clinical Trials. Horm Metab Res. 2017; 49(3):174-179. doi: 10.1055/s-0043-103573.

53. Naderpoor N, Shorakae S, Abell SK, Mousa A, Joham AE, Moran LJ, et al. Bioavailable and free 25-hydroxyvitamin D and vitamin D binding protein in polycystic ovary syndrome: Relationships with obesity and insulin resistance. J Steroid Biochem Mol Biol. 2018; 177:209-215. doi: 10.1016/j. jsbmb.2017.07.012.

54. Hahn S, Haselhorst U, Tan S, Quadbeck B, Schmidt $\mathrm{M}$, Roesler S, et al. Low serum 25-hydroxyvitamin D concentrations are associated with insulin resistance and obesity in women with polycystic ovary syndrome. Exp Clin Endocrinol Diabetes. 2006; 114(10):577-583. doi: 10.1055/s-2006-948308.

55. Mishra S, Das AK, Das S. Hypovitaminosis D and Associated Cardiometabolic Risk in Women with PCOS. J Clin Diagn Res. 2016; 10(5):BC01-4. doi: 10.7860/JCDR/2016/19407.7771.

56. Wehr E, Pieber TR, Obermayer-Pietsch B. Effect of vitamin D3 treatment on glucose metabolism and menstrual frequency in polycystic ovary syndrome women: a pilot study. J Endocrinol Invest. 2011; 34(10):757-763. doi: 10.3275/7748.

57. Panidis D, Balaris C, Farmakiotis D, Rousso D, Kourtis A, Balaris V, et al. Serum parathyroid hormone concentrations are increased in women with polycystic ovary syndrome. Clin Chem. 2005; 51(9):1691-1697. doi: 10.1373/clinchem.2005.052761.

58. Santos BR, Lecke SB, Spritzer PM. Apa-I polymorphism in VDR gene is related to metabolic syndrome in polycystic ovary syndrome: a cross-sectional study. Reprod Biol Endocrinol. 2018; 16(1):38. doi: 10.1186/ s12958-018-0355-9.

59. Ranjzad F, Mahban A, Shemirani AI, Mahmoudi T, Vahedi M, Nikzamir A, et al. MR. Influence of gene variants related to calcium homeostasis on biochemical parameters of women with polycystic ovary syndrome. J Assist Reprod Genet. 2011; 28(3):225-232. doi: 10.1007/s10815-010-9506-4.

60. Wehr E, Trummer O, Giuliani A, Gruber HJ, Pieber TR, Obermayer-Pietsch B. Vitamin D-associated polymorphisms are related to insulin resistance and vitamin D deficiency in polycystic ovary syndrome. Eur J Endocrinol. 2011; 164(5):741-749. doi: 10.1530/ EJE-11-0134.

61. Thys-Jacobs S, Donovan D, Papadopoulos A, 


\section{SUPLEMENTACIÓN DE VITAMINA D EN EMBARAZADAS \\ Y EN MUJERES CON PATOLOGÍAS GINECOLÓGICAS}

Sarrel P, Bilezikian JP. Vitamin D and calcium dysregulation in the polycystic ovarian syndrome. Steroids. 1999; 64(6):430-435. doi: 10.1016/s0039$128 \times(99) 00012-4$.

62. Karadağ C, Yoldemir T, Yavuz DG. Effects of vitamin D supplementation on insulin sensitivity and androgen levels in vitamin-D-deficient polycystic ovary syndrome patients. J Obstet Gynaecol Res. 2018; 44(2):270-277. doi: $10.1111 /$ jog.13516.

63. Selimoglu H, Duran C, Kiyici S, Ersoy C, Guclu M, Ozkaya $\mathrm{G}$, et al. The effect of vitamin D replacement therapy on insulin resistance and androgen levels in women with polycystic ovary syndrome. J Endocrinol Invest. 2010; 33(4):234-238. doi: 10.1007/ BF03345785.

64. Asemi Z, Foroozanfard F, Hashemi T, Bahmani F, Jamilian M, Esmaillzadeh A. Calcium plus vitamin D supplementation affects glucose metabolism and lipid concentrations in overweight and obese vitamin $\mathrm{D}$ deficient women with polycystic ovary syndrome. Clin Nutr. 2015; 34(4):586-592. doi: 10.1016/j. clnu.2014.09.015.

65. Rashidi B, Haghollahi F, Shariat M, Zayerii F. The effects of calcium-vitamin D and metformin on polycystic ovary syndrome: a pilot study. Taiwan J Obstet Gynecol. 2009; 48(2):142-147. doi: 10.1016/ S1028-4559(09)60275-8.

66. Rahimi-Ardabili H, Pourghassem Gargari B, Farzadi L. Effects of vitamin D on cardiovascular disease risk factors in polycystic ovary syndrome women with vitamin D deficiency. J Endocrinol Invest. 2013; 36(1):28-32. doi: 10.3275/8303.

67. Raja-Khan N, Shah J, Stetter CM, Lott ME, Kunselman AR, Dodson WC, et al. High-dose vitamin D supplementation and measures of insulin sensitivity in polycystic ovary syndrome: a randomized, controlled pilot trial. Fertil Steril. 2014; 101(6):1740-1746. doi: 10.1016/j.fertnstert.2014.02.021.

68. Seyyed Abootorabi M, Ayremlou P, BehrooziLak T, Nourisaeidlou S. The effect of vitamin D supplementation on insulin resistance, visceral fat and adiponectin in vitamin $\mathrm{D}$ deficient women with polycystic ovary syndrome: a randomized placebocontrolled trial. Gynecol Endocrinol. 2018; 34(6):489494. doi: 10.1080/09513590.2017.1418311.

69. Ott J, Wattar L, Kurz C, Seemann R, Huber JC, Mayerhofer K, et al. Parameters for calcium metabolism in women with polycystic ovary syndrome who undergo clomiphene citrate stimulation: a prospective cohort study. Eur J Endocrinol. 2012; 166(5):897-902. doi: 10.1530/EJE-11-1070.

70. Asadi M, Matin N, Frootan M, Mohamadpour J, Qorbani M, Tanha FD. Vitamin D improves endometrial thickness in PCOS women who need intrauterine insemination: a randomized double-blind placebo-controlled trial. Arch Gynecol Obstet. 2014; 289(4):865-870. doi: 10.1007/s00404-013-3055-x.

71. Drakopoulos P, van de Vijver A, Schutyser V, Milatovic $\mathrm{S}$, Anckaert E, Schiettecatte J, et al. The effect of serum vitamin D levels on ovarian reserve markers: a prospective cross-sectional study. Hum Reprod. 2017; 32(1):208-214. doi: 10.1093/humrep/dew304.

72. Irani M, Merhi Z. Role of vitamin D in ovarian physiology and its implication in reproduction: a systematic review. Fertil Steril. 2014; 102(2):460-468. e3. doi: 10.1016/j.fertnstert.2014.04.046.

73. Johnson LE, DeLuca HF. Reproductive defects are corrected in vitamin d-deficient female rats fed a high calcium, phosphorus and lactose diet. J Nutr. 2002; 132(8):2270-2273. doi: 10.1093/jn/132.8.2270.

74. Aleyasin A, Hosseini MA, Mahdavi A, Safdarian L, Fallahi P, Mohajeri MR, et al. Predictive value of the level of vitamin D in follicular fluid on the outcome of assisted reproductive technology. Eur J Obstet Gynecol Reprod Biol. 2011; 159(1):132-137. doi: 10.1016/j. ejogrb.2011.07.006.

75. Ozkan S, Jindal S, Greenseid K, Shu J, Zeitlian G, Hickmon C, et al. Replete vitamin D stores predict reproductive success following in vitro fertilization. Fertil Steril. 2010; 94(4):1314-1319. doi: 10.1016/j. fertnstert.2009.05.019.

76. Rudick B, Ingles S, Chung K, Stanczyk F, Paulson R, Bendikson $\mathrm{K}$. Characterizing the influence of vitamin D levels on IVF outcomes. Hum Reprod. 2012; 27(11):3321-3327. doi: 10.1093/humrep/des280.

77. Rudick BJ, Ingles SA, Chung K, Stanczyk FZ, Paulson RJ, Bendikson KA. Influence of vitamin D levels on in vitro fertilization outcomes in donor-recipient cycles. Fertil Steril. 2014; 101(2):447-452. doi: 10.1016/j. fertnstert.2013.10.008.

78. Firouzabadi RD, Rahmani E, Rahsepar M, Firouzabadi MM. Value of follicular fluid vitamin D in predicting the pregnancy rate in an IVF program. Arch Gynecol Obstet. 2014; 289(1):201-206. doi: 10.1007/s00404-013-2959-9.

79. Anifandis GM, Dafopoulos K, Messini CI, Chalvatzas N, Liakos N, Pournaras S, et al. Prognostic value of follicular fluid $25-\mathrm{OH}$ vitamin $\mathrm{D}$ and glucose levels in 
the IVF outcome. Reprod Biol Endocrinol. 2010; 8:91. doi: 10.1186/1477-7827-8-91.

80. van de Vijver A, Drakopoulos P, Van Landuyt L, Vaiarelli A, Blockeel C, Santos-Ribeiro S, et al. Vitamin $\mathrm{D}$ deficiency and pregnancy rates following frozenthawed embryo transfer: a prospective cohort study. Hum Reprod. 2016; 31(8):1749-1754. doi: 10.1093/ humrep/dew107.

81. Polyzos NP, Anckaert E, Guzman L, Schiettecatte J, Van Landuyt L, Camus M, et al. Vitamin D deficiency and pregnancy rates in women undergoing single embryo, blastocyst stage, transfer (SET) for IVF/ICSI. Hum Reprod. 2014; 29(9):2032-2040. doi: 10.1093/ humrep/deu156.

82. Franasiak JM, Molinaro TA, Dubell EK, Scott KL, Ruiz AR, Forman EJ, et al. Vitamin D levels do not affect IVF outcomes following the transfer of euploid blastocysts. Am J Obstet Gynecol. 2015; 212(3):315. e1-6. doi: 10.1016/j.ajog.2014.09.029.

83. Pacis MM, Fortin CN, Zarek SM, Mumford SL, Segars JH. Vitamin D and assisted reproduction: should vitamin D be routinely screened and repleted prior to ART? A systematic review. J Assist Reprod Genet. 2015; 32(3):323-335. doi: 10.1007/s10815-014-0407-9.

84. Braegger C, Campoy C, Colomb V, Decsi T, Domellof $\mathrm{M}$, Fewtrell $\mathrm{M}$, et al.; ESPGHAN Committee on Nutrition. Vitamin D in the healthy European paediatric population. J Pediatr Gastroenterol Nutr. 2013; 56(6):692-701. doi: 10.1097/MPG.0b013e31828f3c05.

85. Collins A. Evaluation and treatment of vitamin D deficiency. Nurse Pract. 2016; 41(11):1-6. doi: 10.1097/01.NPR.0000502797.81231.c6.

86. Marques-Vidal P, Rousi E, Paccaud F, Gaspoz JM, Theler JM, Bochud M, et al. Dietary Intake according to Gender and Education: A Twenty-Year Trend in a Swiss Adult Population. Nutrients. 2015; 7(11):95589572. doi: 10.3390/nu7115481.
87. Cashman KD, Kiely M. Contribution of nutrition science to the vitamin D field-Clarity or confusion? J Steroid Biochem Mol Biol. 2019; 187:34-41. doi: 10.1016/j.jsbmb.2018.10.020.

88. McKenna MJ, Murray BF. Vitamin D dose response is underestimated by Endocrine Society's Clinical Practice Guideline. Endocr Connect. 2013; 2(2):87-95. doi: 10.1530/EC-13-0008.

89. Javed A, Vella A, Balagopal PB, Fischer PR, Weaver AL, Piccinini F, et al. Cholecalciferol supplementation does not influence $\beta$-cell function and insulin action in obese adolescents: a prospective double-blind randomized trial. J Nutr. 2015; 145(2):284-290. doi: 10.3945/jn.114.202010.

90. He C, Lin Z, Robb SW, Ezeamama AE. Serum Vitamin D Levels and Polycystic Ovary syndrome: A Systematic Review and Meta-Analysis. Nutrients. 2015; 7(6):4555-4577. doi: 10.3390/nu7064555.

91. Abrahamsen B, Harvey NC. Vitamin D supplementation for musculoskeletal health outcomes in adults - The end of the beginning? Maturitas. 2019; 122:87-88. doi: 10.1016/j.maturitas.2018.10.011.

92. Rosas-Peralta M, Holick MF, Borrayo-Sánchez G, Madrid-Miller A, Ramírez-Árias E, Arizmendi-Uribe E. Dysfunctional immunometabolic effects of vitamin D deficiency, increased cardiometabolic risk. Potential epidemiological alert in America? Endocrinol Diabetes Nutr. 2017; 64(3):162-173. English, Spanish. doi: 10.1016/j.endinu.2016.11.009.

93. Wang TJ. Vitamin D and Cardiovascular Disease. Annu Rev Med. 2016; 67:261-272. doi: 10.1146/annurevmed-051214-025146. 